\title{
Profile Physical and Phenolic-Chemical of Kumquat Influenced by the Environment Analyzed in Fresh
}

\author{
Soraya Mercedes Pérez ${ }^{1}$ \\ 1 Faculty of Engineering, Natural Resources and Chemical Engineering Department, Tafila Technical University, \\ Tafila P.O. Box 66110, Jordan \\ e-mail: sorayamercedes@yahoo.com
}

\begin{abstract}
Fortunella margarita is an excellent source of acids, sugars and phenolic compounds, the cultivation of which has become popular in Jordan in the last few years. The aim was to observe the quality of the kumquat fruits cultivated in Irbid and the Ghor Valley. Physical properties such as size, density, sphericity, porosity and color were measured. Total titrable acidity (TTA), total soluble solids (TSS), Ascorbic Acid (AA), pH and phenolic compounds were evaluated in the pulp-rind extract. Total phenols (TPC), flavonoids and carotenoids were measured by spectrophotometry at 765, 430 and 470-664 nm, respectively. High porosity (67\%), fruit size (37.4 mm) and juice yield $(25.5 \%)$ were achieved in the Ghor kumquat crop. The high levels of TPC (2039.2 mg GAE/100 g), AA $(0.361 \mathrm{mg} / \mathrm{ml})$ and carotenoids $(1.15 \mathrm{mg} / 100 \mathrm{~g})$ registered in this same crop, attribute it better functional and medicinal properties. Moreover, the Ghor cultivation presented better taste, associated with high values of $\mathrm{pH}(3.47)$, TSS (5\%) and low levels of TTA (1.55\%). The high concentration of total carotenoids recorded in the Ghor's kumquat was associated with the intense and bright orange color of its rind, related with high values of the color coordinates $+\mathrm{a},+\mathrm{b}$ and $\mathrm{L}$ of the CIELab system. The great porosity of its rind, makes the kumquat a suitable fruit for impregnating with salt or sugar solutions as well as developing new kumquat products with better flavor and nutritional value. The ranges found in the properties of kumquat crops, are attributed to the different temperatures of Ghor and Irbid.
\end{abstract}

Keywords: Fortunella margarita, carotenoids, kumquat, CIELab color, TPC.

\section{INTRODUCTION}

The kumquat fruits are citrus native to China, which propagated to other regions by Robert Fortune who transported them to Europe in 1846 [Spiegel-Roy \& Goldschmidt, 1996] and from there they have spread all around the world. The kumquat belongs to the Rutacea family and it is known for its sweet and sour taste, small size and consumable rind [www. theplantlist.org/tpl/record/kew-2724150]. It has four major cultivated types: Fortunella Japonica, Fortunella margarita, Fortunella crassifolia and Fortunella handsii [Ogawa et al., 2001]. Dugo et al., 2010; inserted to the classification the variety Fortunella polyandra "Malayan kumquat", naming Fortunella Japonica (Thunb) as Swingle "Marumi kumquat" and Fortunella margarita (Lour) as Swingle "Nagami kumquat". Other authors include varieties as the Nordmann Seedless Kumquat, which is similar size and flavor to Nagami; the Fukushu Kumquat which is a hybrid kumquatmandarin and the Centennial Variegated Kumquat [www.fourwindsgrowers.com/blogs/fourwinds-growing/8-great-kumquat-varieties].

The variety Fortunella margarita also called "Nagami kumquat" is the most popular species worldwide [Babazadeh \& Jaimand, 2019], especially in the USA. The fruit of this variety stands out for its medium, oval size fruit with a bright orange, thick peel as well as a sweet-tart pulp and juice.

Kumquat is an excellent source of sugars and acids. Some researchers have claimed that the variety may influence the physicochemical traits of kumquat [Tang et al., 2013]. 
Among the components of kumquat are the soluble solids (TSS), which are a mixture of sugars and organic acids. The quotient of TSS per titratable total acidity (TTA) displays the Maturity Index (MI), which associates the taste quality. Sugars represent around $80 \%$ of TSS, sucrose being the most plentiful sugar, followed by fructose and glucose [Varnam \& Sutherland, 2012].

Ascorbic acid, present in considerable quantities in the kumquat, is a compound with great antioxidant power that the human body is not able to synthesize, for this reason; this is taken from vegetables and fruits. This acts as an active antioxidant in oxidation and reduction reactions, associating the content of ascorbic acid to the antioxidant capacity of fruits and vegetables [Pérez, 2020]. Color is the result of chemical, biochemical, physical and even microbial processes ocurring during the growth, at maturity- ripening stage and post-harvest management of the fruit. It is considered a quality attribute, which combined with sweetness, acidity, aroma and size, contribute to consumer acceptability.

Some health benefits of kumquat fruit are due to its "functional" properties attributed to its phenolic compounds, such as the total phenols (TPC), flavonoids, carotenoids, etc. The "functional" qualities are understood as those that make a food have a potentially positive effect on health beyond basic nutrition [Pérez, 2004; Pérez, 2014]. The TPC are antioxidant organic compounds, with variable molecular structures, which contain at least one phenol group, and an aromatic ring linked to a hydroxyl group. They comprise a diverse group of secondary metabolites present in higher plants [Labbe et al., 2016]. Carotenoids are natural pigments known in some research as promoters in the reduction of cancers, cataracts and heart disease [Preedy et al., 2011].

There is no decisive information regarding when the cultivation of kumquat started in Jordan. However, it is clear that until a few years ago, the exportation of kumquat fruits from Jordan was absent. Statistics of the Ministry of Agriculture show that until 2014 the exported quantity of kumquat was zero. In 2015 Jordan exported about 23 tons of kumquat and in next year the exported quantity show some increment [USAID, 2018]. This light increasing of export volume suggests that there is a possibility of a future expansion of growing Kumquat trees [Jordan Investment Commission, 2017].
The main goal of this research was to observe the quality parameters of the Fortunella margari$t a$ variety kumquat cultivated in two different locations in Jordan, through the evaluation of some physical, chemical and color traits.

\section{MATERIALS AND METHODS}

The kumquat fruits samples for the actual study were collected at full ripening stage. The fruits of Lot 1 belong to a particular home garden in the Irbid governorate, where they have received common cares by common citizen with common knowledge of agricultural practice. The samples of Lot 2 were grown under a professional technical care in plant nursery, at the Ghor valley. Behind the difference in agricultural practices between the two cites the temperature is also quite different. In the Ghor area the temperature almost never falls to zero Celsius. In contrast in the location of Lot 1 , the temperature falls to zero several times between December and February. Generally, the temperature of lot 1 location is 3 to 5 Celsius degrees less than the Lot 2 location [Pérez, 2020]. The experimental design was completely randomized with three replicates per analysis. The physical parameters measured corresponded to the whole fruit. The chemical-phenolic characterization was evaluated in the extract "rind-pulp mixture" considering that kumquat is a fruit that is preferably consumed natural, whole and with peel. The color was evaluated in the rind of the fruit.

\section{Physical analysis}

The fruits were individually weighed by an electronic balance, model Metler AE100. The three main dimensions length, width, and thickness were measured by a digital caliper. The bulk and real density was measured using the methods followed for Jaliliantabar et al. (2012) and Rafiee et al. (2007) for Irani kumquat and bergamot respectively. The sphericity was determined by the quotient $\Phi=D / L$ [Topuz et al., 2004]. The values of sphericity close to one represent major spherical shape of the fruit.

The color was measured by a Minolta model CR-410 colorimeter. The measurements were performed at four different points on the rind samples. The color was assigned according to the CIELab system, which uses Cartesian coordinates" $\mathrm{L}, \mathrm{a}, \mathrm{b}$ " to calculate the color in a space. The space where the parameter " $a$ " moves between 
green and red, determines $+\mathrm{a}$ degree of redness and $-\mathrm{a}$ the degree of greenness. The parameter "b" moves between blue and yellow where the -b coordinate indicates the degree of blueness and $+b$ the degree of yellowness. The luminosity "L" represents the degree of clarity of the sample, moving between $\mathrm{L}=0$ (black, maximum darkness) and $\mathrm{L}=100$ (white, maximum clarity). Chroma" C" describes the intensity or purity of the color (maximum saturation level) and the dullness of the color (minimum saturation level). This diagram is showed in the Figure 1. Chroma $C$, is calculated by the expression:

$$
C=\left(a^{2}+b^{2}\right)^{1 / 2}
$$

Color was also measured using the CIELCh polar coordinates system, which, also uses the Chroma " $C$ ". It includes the hue angle $h^{*}$, which describes the predominant color that an observer perceives. This is calculated by the expression

$$
h^{*}=\arctan (b / a)
$$

Where the angle $h^{*}$ associates the following references: $0^{\circ}=$ red-purple, $90^{\circ}=$ yellow, $180^{\circ}=$ bluish-green and $270^{\circ}$ blue [Manera et al., 2012], illustrated in the Figure 1. The difference of color $(\Delta E)$ is calculated using the equation:

$$
\Delta E=\left(\Delta L^{2}+\Delta a^{2}+\Delta b^{2}\right)^{1 / 2}
$$

\section{Chemical-phenolic analysis}

Total titratable acidity (TTA) was evaluated by titration with sodium hydroxide $0.1 \mathrm{~N}$ and displayed as percentage of citric acid [AOAC, 1980]. The $\mathrm{pH}$ values were determined using a Ingold U402-57/120 digital $\mathrm{pH}$ meter. The total soluble solids (TSS) were measured using a ABBE Carl

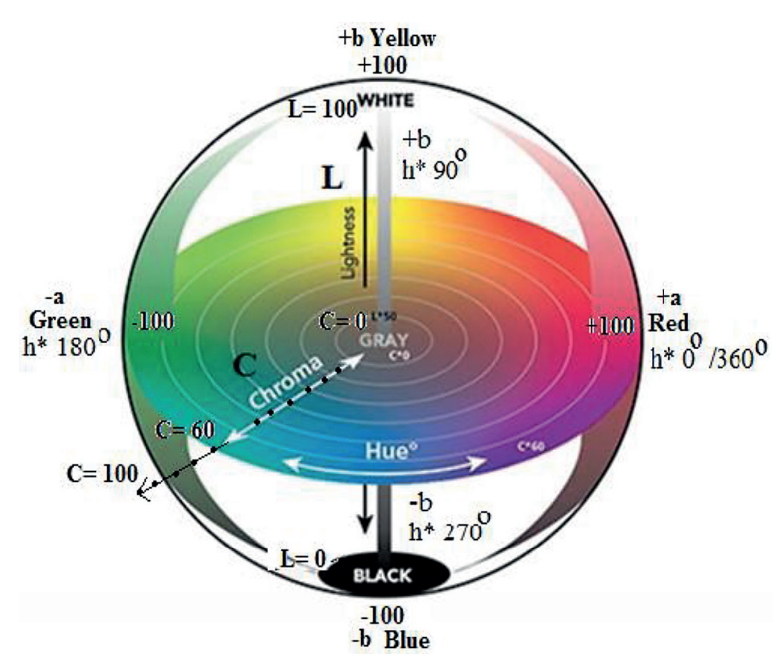

Fig. 1. CIELab and CIELCh systems in the space (Modified after Pérez, 2020)
Zeiss model 89553 refract meter. The results were reported as Brix degrees at $25{ }^{\circ} \mathrm{C}$. The maturity index (IM) was calculated by the quotient IM = TTS/TTA.

The concentration of total polyphenols (TPC) was determined by using the Folin-Ciocalteu method [Makkar, 2000], reporting the results as mg. Gallic acid (mg GAE)/100 g sample. This method is based on the treatment of diluted samples with the Folin-Ciocalteu reagent and aliquots of $20 \%$ sodium carbonate solution, with subsequent measurement of the absorbance at $765 \mathrm{~nm}$.

The total flavonoids content was quantified using one the methods followed by Shyi-Neng \& Chi-Tang (2016), which is based on the chelation technique with $\mathrm{AlCl}_{3}$. An aliquot of kumquat extract was mixed with $2 \%$ methanolic $\mathrm{AlCl}_{3} \cdot 6 \mathrm{H}_{2} \mathrm{O}$. The mixture was at rest at room temperature over 10 minutes. Then the absorbance was measured to $430 \mathrm{~nm}$. The standard curve was made by standards of quercetin solutions. The total flavonoids content was reported as $\mathrm{mg}$. quercetin equivalents $/ 100$ dry extract using the calibration curve obtained previously.

The ascorbic acid (vitamin C) content was determined following the official method AOAC 967.21 [AOAC 2000]. It was carried out by volumetric titration with 2,6-Dichloroindophenol. The indicator was standardized with a standard solution of L-ascorbic acid 750 ppm (ac. L-ascorbic with metaphosphoric acid $\mathrm{H}_{3} \mathrm{PO}$, conc. 5\%).

Total carotenoids were determined following the method presented by Van Wyka et al. (2009). The kumquat rinds were dried and frozen at $-56^{\circ} \mathrm{C}$ for 4 days to eliminate any trace of moisture. Subsequently, the rind samples were ground and frozen at $-80{ }^{\circ} \mathrm{C}$ until the moment of analysis. The complete process was carried out under low light conditions to reduce photo destruction. A mixture of ethanol, butylated, hydroxyl toluene and diethyldithiocarbamate was mixed with $0,2 \mathrm{~g}$ of frozen sample and carried to spectrophotometry with wavelengths corresponding to 470,649 and $664 \mathrm{~nm}$.

\section{Statistical analysis}

Three replicates were taken from each sample and the results were used for statistical analysis. The values were reported as mean $\pm \mathrm{SD}$ for all variables presented. Statistical t-student analysis was used to check the significant differences $p$ $<0.05$. In general, the values registered for both lots revealed a significant difference between the measured parameters. 


\section{RESULTS AND DISCUSSION}

The physicochemical parameters evaluated for the two kumquat crops considered in this study are presented in the Table 1 . Three replicates for each sample were represented as mean \pm standard deviation. L1: kumquat samples from Irbid; L2: kumquat samples from the Ghor valley

The results of the physical analysis indicated that the fruits of Lot 2 had superior size (37.4 $\mathrm{mm})$, juice yield $(25 \%)$ and density $(1.23 \mathrm{~g} /$ $\left.\mathrm{cm}^{3}\right)$. The values of sphericity closer to the unit registered in lot $1(\varepsilon=77.02 \%)$ indicate a better spherical shape for the fruits of this crop; in contrast with Lot 2 , which obtained lower values of sphericity $(\varepsilon=74.19 \%)$ in harmony with the oval shape seen its fruit. Jaliantabar et al. (2012) presented the studies of the physical properties of kumquat (var. Fortunella margarita) from Iran. They reported the values in the same order as Lot 1 , but differing notably from the values for Lot 2 .

The high values of porosity $(67 \%)$ found at Lot 2, even higher than the orange fruit (19\%), leads to think that they would have a better response to impregnation with salt or sugar solutions [Pérez, 2004; Cháfer et al., 2003], which would allow the development of kumquat products with better taste and nutritional value.

The kumquat fruits of both lots, were evaluated at full ripening stage, reaching their maximum levels of sweetness. Lot 2 registered a highest content of soluble solids (TSS $=5 \%$ ), $\mathrm{pH}$ (3.47) and lowest percentage of titratable acidity $(\mathrm{TTA}=1.55)$, associated with a high maturity in$\operatorname{dex}(\mathrm{IM}=3.22)$. The TTA values are reported as percentage of citric acid, following the analysis presented by Babazadeh \& Jaimand (2019). For kumquat variety Fortunella Margarita, the citric acid was the main acid present in this fruit. The high values of TSS, $\mathrm{pH}$ together with the low levels of TTA of lot 2 were associated with the best organoleptic properties of the fruits for fresh consumption. Figure 2 shows a comparative profile between TTA, pH, IM and TTA for kumquat of both lots.

The analyses recorded low flavonoid content (131.5 QE mg/100 g) with high levels of TPC (2039.21 mgGAE/100 g) and total carotenoids $(1.15 \mathrm{mg} / 100 \mathrm{~g})$ for Lot 2 . On the basis of these results, and comparing with the values for Lot1, a tendency to increase the TPC together with the total carotenoids was observed in this analysis; deducing a direct relationship between them. This trend was also observed in studies conducted by Gun-Ae Yoon et al, 2012 for plant foods consumed in Korea [Gun-Ae Yoon et al., 2012].

In relation to the concentration of carotenoids in food, Britton et al. (2009) has proposed the following classification ranges: low $0-0.1 \mathrm{mg} / 100 \mathrm{~g}$; moderate $0.1-0.5 \mathrm{mg} / 100 \mathrm{~g}$; high $0.5-2 \mathrm{mg} / 100$ $\mathrm{g}$; very high $2 \mathrm{mg} / 100 \mathrm{~g}$. According to this scale, the total carotenoids content for both lots, would fall into "high range", although there is a clear margin of difference between them (Lot 1: 0.51 $\mathrm{mg} / 100 \mathrm{~g}$; Lot 2: $1.15 \mathrm{mg} / 100 \mathrm{~g}$ ). Moreover, an inverse relationship was observed between the flavonoid content and MI recorded for each lot (Fig. 2), according with previous studies presented in fruits such as pomegranates from Chile var. Chaca, Wonderful, Codpa [Labbe et al., 2016.] and the pomegranates native to Jordan [Pérez, 2020]. The gradual reduction of the flavonoid content during the maturation period until reaching minimum values at full ripening stage, probably occurs because the flavonoids are consumed during the maturation period to achieve the characteristic "flavor and taste" at full ripening stage. This mechanism is carried out by conversion of the structure of flavonoids as (o) condensed tannin, which is synthesized in other antioxidant forms [anthocyanins synthase (AS) and 3-glycosyl transferase $(3 \mathrm{GT})]$, through an increase

Table 1. Results of the traits analyzed in fruits of kumquat var. Fortunella margarita

\begin{tabular}{|c|c|c|c|c|c|c|c|c|c|}
\hline \multicolumn{10}{|c|}{ Physical } \\
\hline Lot trait & $\begin{array}{l}\text { Length } \\
(\mathrm{mm})\end{array}$ & $\begin{array}{l}\text { Width } \\
(\mathrm{mm})\end{array}$ & $\begin{array}{l}\text { Thickness } \\
(\mathrm{mm})\end{array}$ & $\begin{array}{l}\text { Mass } \\
\text { (g) }\end{array}$ & $\begin{array}{l}\rho_{\text {real }} \\
3 \\
\end{array}$ & Sphericity & $\begin{array}{c}\begin{array}{c}\text { Volume } \\
(\mathrm{ml})\end{array} \\
\end{array}$ & \% Juice & Porosity \\
\hline L1 & $1.05 \pm 1.1$ & $25.1 \pm 2.3$ & $24.03 \pm 1.5$ & $10.89 \pm 1.8$ & $1.18 \pm 0.1$ & $77.02 \pm 2.3$ & $9.22 \pm 0.8$ & $20.5 \pm 1.5$ & $59.12 \pm 2.1$ \\
\hline L2 & $37.4 \pm 0.9$ & $27.8 \pm 1.8$ & $21.14 \pm 1.2$ & $13.17 \pm 2.1$ & $1.23 \pm 0.1$ & $74.19 \pm 1.7$ & $10.70 \pm 1.1$ & $25.5 \pm 1.9$ & $67.01 \pm 1.7$ \\
\hline \multicolumn{10}{|c|}{ Chemical } \\
\hline Lot trait & $\mathrm{pH}$ & \%TTA & ${ }^{\circ}$ Brix & TPC & Flavonoids & IM & AA & $\begin{array}{c}\text { Total } \\
\text { carotenoids }\end{array}$ & $\begin{array}{l}\text { Refractive } \\
\text { index }\end{array}$ \\
\hline L1 & $3.18 \pm 0.01$ & $1.95 \pm 0.04$ & $4.5 \pm 0.2$ & $1622.5 \pm 1.1$ & $165.5 \pm 0.6$ & $2.3 \pm 1.1$ & $0.33 \pm 0.4$ & $0.51 \pm 0.3$ & $1.3401 \pm 1.2$ \\
\hline L2 & $3.47 \pm 0.02$ & $1.55 \pm 0.02$ & $5.0 \pm 0.1$ & $2039.2 \pm 0.9$ & $131.5 \pm 0.4$ & $3.22 \pm 0.9$ & $0.361 \pm 0.7$ & $1.15 \pm 0.8$ & $1.3403 \pm 0.8$ \\
\hline
\end{tabular}




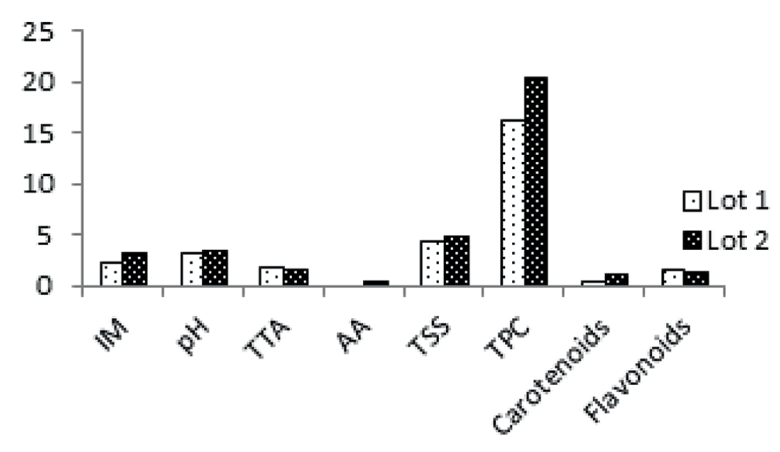

Fig. 2. Phenolic-chemical parameters in kumquat fruits. Lot 1: Irbid samples; Lot 2: Ghor valley samples

in enzymatic activity until achieve the ripening stage [Cristosto et al., 2000].

A high content of ascorbic acid was recorded for Lot 2 ( $\mathrm{AA}=0.36 \mathrm{mg} / \mathrm{lit})$ together with the high TPC values. Possibly this behavior is due to the nature of the ascorbic acid. AA is an active antioxidant that creates metabolites that increase the TPC levels, due to the functional similarities between them [Kulkarni \& Aradhya, 2005]. The ascorbic acid content found in both lots was lower than the values reported by Babazadeh et al, 2019; for kumquat (Fortunella margarita) on Citrus Rootstocks.

The high concentration of carotenoids in the kumquat of Lot 2, was associated with the presence of a remarkable orange color in the fruit rind. The maximum levels of TPC together with the high concentration of carotenoids, suggest that this kumquat crop could contribute significantly to the consumption of important antioxidant and bioactive compounds for the population. Similar analyses were discussed by Pinheiro et al., 2019 and Giuffrida et al., 2010 for the kumquat from Brazil and orange juice of different varieties, respectively.

The high content of carotenes present at full ripening stage, considerably defines the color of the fruits. The color is one of the attributes that most influence "the visual quality" of fresh food.
This property was evaluated using the Cartesian "CIELab" and polar "CIELCh" coordinate systems. Table 2 presents the color coordinates for the lots of this study. Four measurements for each three replicates of each sample. Lot 1: kumquat from Irbid; Lot 2: kumquat from the Ghor valley

The results recorded of the color analysis in the rind of the kumquat, revealed high values of the coordinates $+\mathrm{a},+\mathrm{b}$ and $\mathrm{L}$ for Lot 2 . These results are interpreted as colors redder, yellower and brighter than the kumquat fruit of Lot 1 . The product of this combination is the bright orange hue, associated with the high content of total carotenoids that presented the fruits of this lot. These results lead to deduce a possible direct relationship of the maturity degree with the color coordinates red $(+a)$, yellow $(+b)$ and carotene content in a fresh food. Some authors say that the typical color reached at full ripening stage, content and composition of carotenoids in fruits is strongly related by genotype [Roa et al., 2015]. This leads to thinking that the relationship between color and MI could also depend of the variety of fruit. External factors such as: climatic conditions, quality of the farmland, and post-harvest management, could also affect the color and composition of the fruit.

The results monitored of the polar coordinates $h^{*}$ and $C$, which represent the hue and purity of color respectively, in general, presented a direct relationship with the coordinates $\mathrm{L}$ and $\mathrm{b}$ for both lots. The high values of $C$ and $h^{*}$ achieved for the kumquat rinds of Lot 2, placed the points on the orange region of the color diagram compared to the yellow hue recorded for Lot 1 (Fig. 3).

The total color difference was evaluated using the numerical parameter $\Delta E$. Pankaj et al. (2013) and Adekunte et al. (2010); classify the color difference $\Delta E$ as very different $\Delta E>3$; different 1.5 $<\Delta E<3$ and small difference $1.5<\Delta E$. According to this criterion and based on the registered color coordinates, the result obtained $\Delta E=24.50$ would

Table 2. Coordinates of color CIELab and CIELCh for the rind of kumquat fruits

\begin{tabular}{|c|c|c|c|c|c|c|c|c|c|}
\hline \multicolumn{9}{|c|}{ Lot 1} & \multicolumn{5}{c|}{ Lot 2} \\
\hline $\mathrm{L}$ & $\mathrm{a}$ & $\mathrm{b}$ & $\mathrm{C}$ & $\mathrm{h}$ & $\mathrm{L}$ & $\mathrm{a}$ & $\mathrm{b}$ & $\mathrm{C}$ & $\mathrm{h}$ \\
\hline $52.45 \pm 1.10$ & $13.21 \pm 0.20$ & $40.05 \pm 1.22$ & $38.545 \pm 0.16$ & $69.50 \pm 1.09$ & $62.45 \pm 0.20$ & $40.15 \pm 1.10$ & $55.05 \pm 1.10$ & $68.45 \pm 0.66$ & $53.97 \pm 1.80$ \\
\hline $58.15 \pm 0.90$ & $18.93 \pm 0.34$ & $41.02 \pm 0.93$ & $45.15 \pm 0.90$ & $65.22 \pm 0.94$ & $61.92 \pm 0.33$ & $37.53 \pm 0.74$ & $55.93 \pm 0.90$ & $67.09 \pm 0.97$ & $56.51 \pm 0.19$ \\
\hline $57.27 \pm 1.40$ & $8.57 \pm 0.41$ & $45.32 \pm 0.53$ & $46.17 \pm 1.40$ & $79.47 \pm 1.40$ & $57.33 \pm 1.20$ & $30.27 \pm 0.40$ & $46.77 \pm 0.61$ & $55.57 \pm 1.10$ & $57.32 \pm 0.50$ \\
\hline $55.82 \pm 2.00$ & $14.48 \pm 0.75$ & $48.02 \pm 0.72$ & $50.52 \pm 2.00$ & $73.34 \pm 1.06$ & $56.62 \pm 1.08$ & $35.82 \pm 2.00$ & $44.53 \pm 1.34$ & $56.82 \pm 0.14$ & $51.82 \pm 1.06$ \\
\hline $53.15 \pm 1.40$ & $14.57 \pm 1.40$ & $38.94 \pm 0.84$ & $41.54 \pm 1.40$ & $69.47 \pm 0.44$ & $60.29 \pm 1.12$ & $37.47 \pm 1.40$ & $49.05 \pm 1.77$ & $61.45 \pm 1.01$ & $52.98 \pm 1.88$ \\
\hline
\end{tabular}



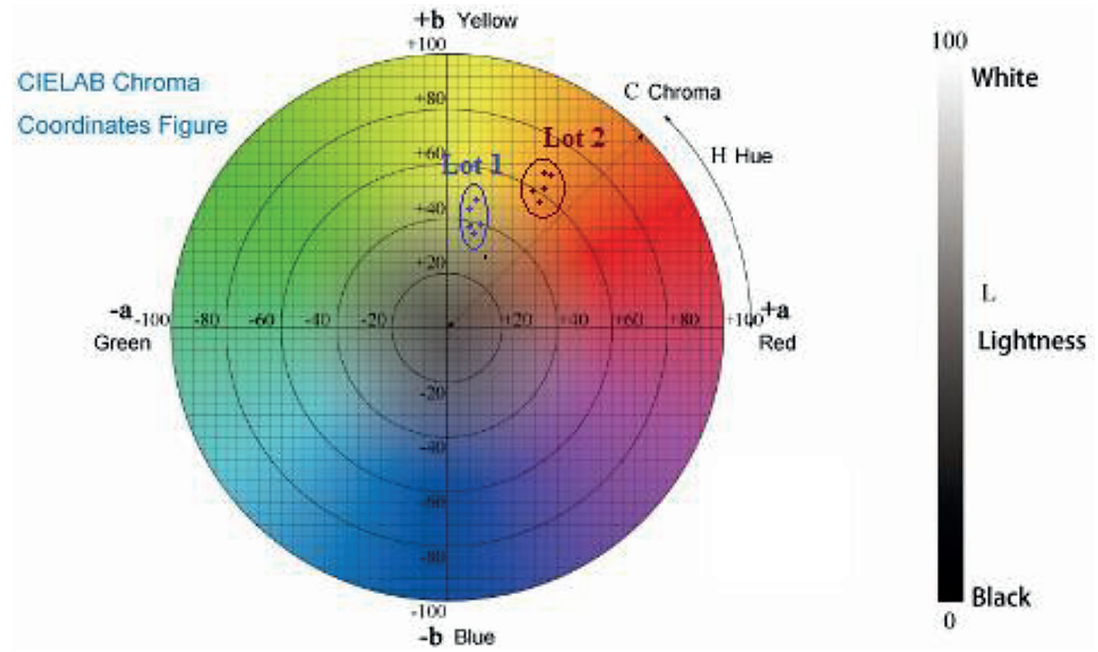

Fig. 3. Plane of CIELab and CIElCh coordinates for rind of kumquat fruits, (Modified after Pérez, 2020) Lot1: Irbid samples; Lot 2: Ghor valley samples

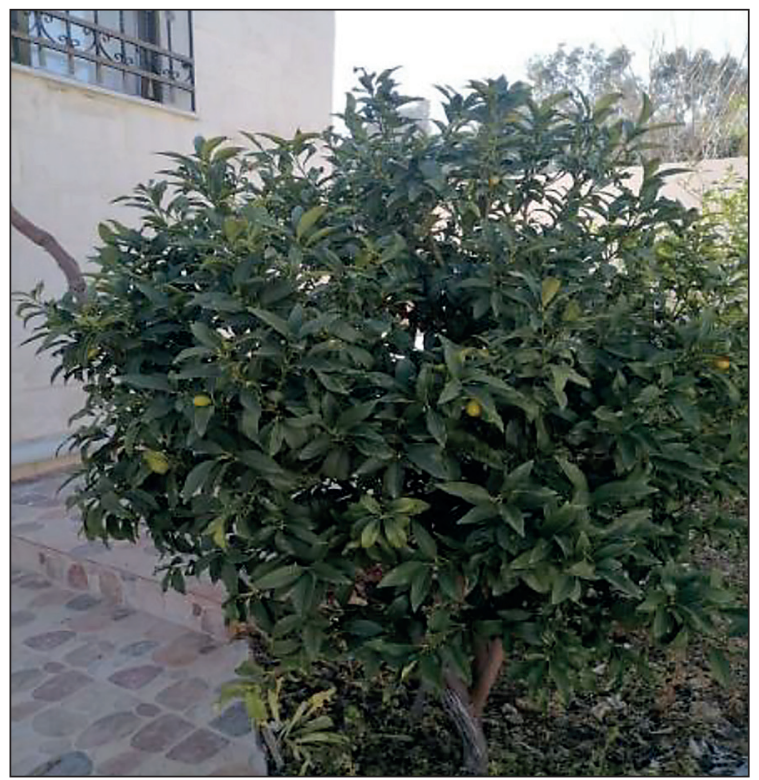

Fig. 4. Kumquat tree in a particular home garden (Irbid location)

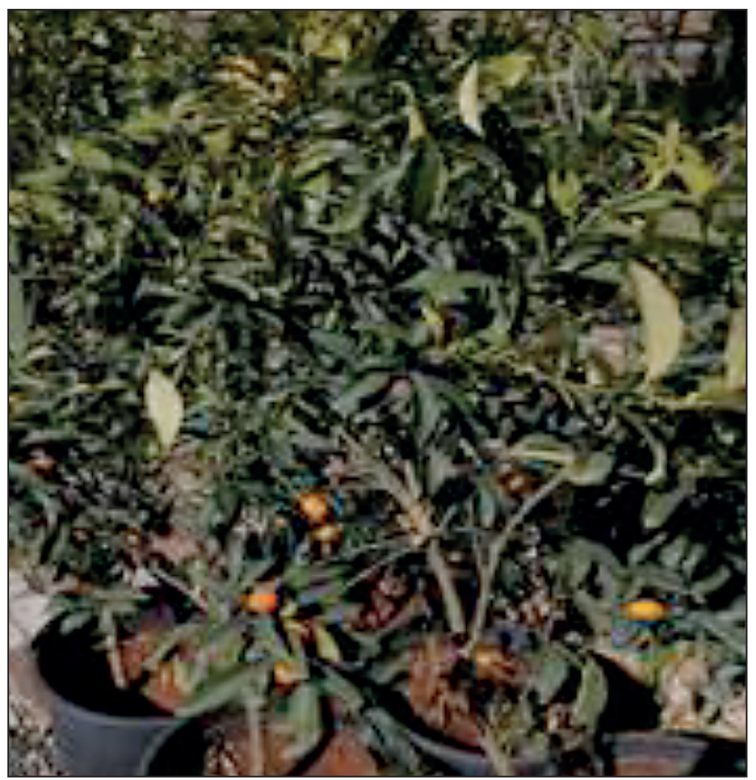

Fig. 5. Kumquat tree of plant nursery (Ghor valley)

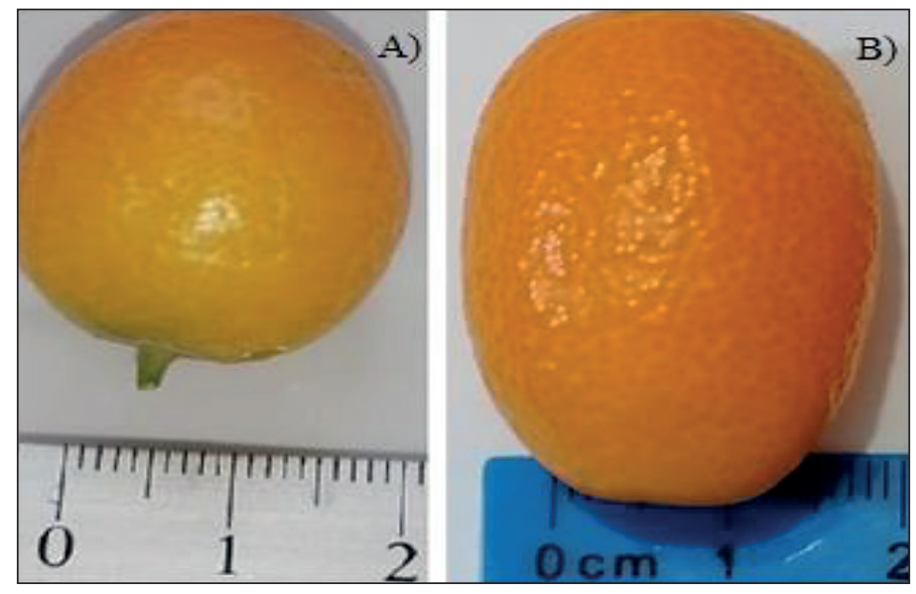

Fig. 6. Kumquat fruit from Irbid location (A) and Ghor Valley (B) 
reveal a great color difference between both lots. The kumquat trees are shown in the Figs. 4 and 5. They show that the fruits of home garden are of smaller size and have more yellow color than the plant nursery (Fig. 6).

\section{CONCLUSIONS}

The results of the actual study lead to conclude that there is a possibility of a future expansion of growing kumquat trees in Jordan. Although the results indicate that the cultivation in green houses is better, the kumquat production in open spaces as at home gardens, under normal and unprofessional care practices also produces fruits of acceptable quality.

The kumquat fruits from Ghor (plant nursery), presented lesser acidity, greater size, more sweetness, higher phenolic content and better color than the fruit from Irbid location (home garden). This clear difference may be partially due to the better irrigation and fertilization techniques that the fruit receive in the plant nursery. However, the different ranges in phenolic-chemical and physical properties between the fruits of both locations are mostly attributed to the temperatures difference that exists between Ghor area and Irbid location. The Ghor's kumquat in comparison with that of Irbid presents better functional properties due to its higher concentration in TPC, Carotenoids and Ascorbic Acid. The low values of the Flavonoid content registered together with the high Maturity Index of Ghor's kumquat, are associated with the consumption of flavonoids during the maturation stage, which is necessary to improve the taste of the fruit at full ripening stage. The presence of the intense orange color in kumquat from the Ghor valley was clearly related to the highest content of total carotenes present in the fruit rind. Finally, the high porosity of kumquat fruit, between $67 \%$ and $59 \%$, would allow it to be used as a matrix for impregnation with salt or sugar solutions to develop new kumquat products with better flavor and nutritional value.

\section{REFERENCES}

1. Adekunte A., Tiwari B., Cullen P., Scannell A., O'Donnell C. 2010.Effect of sonication on color, ascorbic acid and yeast inactivation in tomato juice. Food Chemistry, 122(3), 500-507.
2. AOAC 1980. No 942.15. Acidity titrable of fruit. Official Methods of Analysis. Association of Official Analytical Chemists, Gaithersburg, MD, USA.

3. AOAC 2000. No 967.21. Ascorbic acid in Vitamin Preparations and Juices. Official Methods of Analysis. 17th ed. Association of Official Analytical Chemists, Gaithersburg, MD, USA.

4. Babazadeh-Darjazi B., Jaimand K. 2019. Physicochemical Characteristics of Kumquat (Fortunella margarita) on Citrus Rootstocks. Journal of Medicinal Plants and By-products, 2, 105-114.

5. Britton G., Pfander H., Liaaen-Jensen F., Khachik S. 2009. Carotenoids in food. In Carotenoids. Springer: Basel, Switzerland.

6. Cháfer M., González-Martínez C., Chiralt A., Fito P. 2003. Microstructure and vacuum impregnation response of citrus peels. Food Research International, 36(1), 35-41.

7. Cristosto C.H., Mitcham E.J., Kader A.A. 2000. Pomegranate: recommendations for maintaining postharvest quality. Produce. Facts. Postharvest Research and Information Centre, University of California, Davis, USA.

8. Dugo G., Mondello L. 2010. Citrus Oils: Composition, Advanced Analytical Techniques, Contaminants and Biological Activity. CRC Press, Taylor and Francis group, BocaRaton.

9. Giuffrida D., Dugo P., Salvo A., Saitta M., Dugo G. Free carotenoid and carotenoid ester composition in native orange juices of different varieties. Fruits, 2010, 65, 277-284.

10. Yoon G., Yeum K.J., Cho Y.S., Oliver Chen C.Y., Tang G., Blumberg J.B., Russell R.M., Sun Yoon and Yang Cha Lee-Kim. 2012. Carotenoids and total phenolic contents in plant foods commonly consumed in Korea. https://www.ncbi.nlm.nih.gov/ pmc/articles/PMC3542437/.

11. https://www.fourwindsgrowers.com/blogs/fourwinds-growing/8-great-kumquat-varieties. (accessed on 1 March 2021).

12. Jaliliantabar F., Lorestani A.N., Gholami R. 2013. Physical properties of kumquat fruit. Int. Agrophys, $27,107-109$.

13. Jordan Investment Commission. 2017. Pre-Feasibility Study Soilless Cultivation Project Zarqa Governorate, 18.

14. Kulkarni A.P., Aradhya S.M. 2005. Chemical changes and antioxidant activity in pomegranate arils during fruit development. Food Chemistry, 93, 319-324. DOI: 10.1016/j. foodchem.2004.09.029

15. Labbe M., Ulloa P., Lopez F., Saenz C., Peña A., Salazar F. 2016. Characterization of chemical compositions and bioactive compounds in juices from pomegranates (Wonderful, Chaca and Codpa) at different maturity stages. Chilean Journal of Agricultural Research, 76(4). 
16. Makkar H.P.S. 2000. Quantification of Tannins in Tree Foliage. A Laboratory Manual for the FAO/IAEA Coordinated Research Project on 'Use of Nuclear and Related Techniques to Develop Simple Tannin Assay for Predicting and Improving the Safety and Efficiency of Feeding Ruminants on the Tanniniferous Tree Foliage'. Joint FAO/IAEA Division of Nuclear Techniques in Food and Agriculture. Vienna, Austria.

17. Manera F.J., Legua P., Melgarejo P., Martínez R., Martínez J.J., Hernández F. 2012. Effect of air temperature on rind colour development in pomegranates. Scientia Horticulturae, 134, 245-247. DOI: 10.1016/j/scienta.2011.11.016

18. Ogawa K., Kawasaki A., Omura M., Yoshida T., Ikoma Y., Yano M. 2001. 3',5'-Di-C-beta-glucopyranosylphloretin, a flavonoid characteristic of the genus Fortunella. Phytochemistry, 57, 737-742.

19. Pathare P.B., Opara U.L., Al-Said F.A. 2013. Colour Measurement and Analysis in Fresh and Processed Foods: A Review. Food Bioprocess Tech., 6, 36-60. DOI: $10.1007 /$ s11947-012-0867-9

20. Pérez S.M. 2004. Aplicación de la deshidratación osmótica para la obtención de rodajas de naranja con procesamiento mínimo. [ Doctoral dissertation]. Politechnic University of Valencia, Spain.

21. Pérez S.M. 2014. Functional food that are adjusted to Ecuadorian nutrional needs, and optimal technical processing. La Tecnica. Ecuador, 13, 74-81.

22. Pérez S.M. 2020. Phenolic-chemical constituents, physical and color characteristics of pomegranates (Punica granatum L.) native to Jordan at full ripening stage. A comparative study. International Journal for Environment \& Global Climate Change. Jordan, 5(2), 27-30. Journal of International Agriculture Environmental Science, 7(5), 1-4.

23. Pinheiro-Sant'A.H., Anunciação P., Silva e Souza C., De Paula Filho G., Salvo A., Dugo G., Giu-rida D. 2019. Quali-Quantitative Profile of Native Carotenoids in Kumquat from Brazil by HPLC-DAD-APCI/MS. Foods, 8, 166. DOI: 10.3390/foods 8050166 .
24. Preedy V.R., Watson R.R., Pate V.B. 2011. Nuts and Seeds in Health and Disease Prevention, Academic Press, London.

25. Rafiee S., Keramat Jahromi M., Jafari A., Sharifi M., Mirasheh R., Mobli H. 2007. Determining some physical properties of bergamot (Citrus medica). Int. Agrophysics., 21, 293-297.

26. Roa A.R., Garcia-Luis A., Barcena J.L.G., Huguet C.M. 2015. Effect of 2,4-D on fruit sugar accumulation and invertase activity in sweet orange cv Salustiana. Aust J Crop Sci., 9, 105-111.

27. Lou S.N., Ho C.T. 2017. Phenolic compounds and biological activities ofsmall-size citrus: Kumquat and calamondin. Journal of Food and Drug Analysis, 25(1), 162-175.

28. Spiegel-Roy P., Goldschmidt E.E. 1996. The Biology of Citrus. Cambridge University Press.

29. Tang CH, Lu MH, Tsai WH. 2013. A new variety of Kumquat (Fortunella crassifolia swingle) tainung No.1 Citrin. J Taiwan Agric Rec., 62, 83-91.

30. The plant list. Online: http://www.theplantlist.org/ tpl/record/kew-2724150 (accessed on 28 February 2021).

31. Topuz A., Topakci M., Canakci M., Akinci I., Ozdemir F. 2004.Physical and nutritional properties of four orange varieties. J Food Eng., 66, 519-523.

32. USAID. 2018. Preliminary Economic Feasibility Study to Establish a Plant for Grading and Juicing Citrus in Tabaquet Fahel-Irbid Governorate, 25.

33. Van-Wyka A.A., Huysamera M., Barry G.H. 2009. Extended low temperature shipping adversely affects rind colour of 'Palmer Navel' sweet orange [Citrus sinensis (L.) Osb.] due to carotenoid degradation but can partially be mitigated by optimising post-shipping holding temperature. Postharvest Biol Technol., 53, 109-116.

34. Varnam A., Sutherland J.M. 2012. Beverages: Technology, Chemistry and Microbiology. Springer Science \& Busines-Media. New Y. 